\title{
Misconceptions and truths for feeding patients in the intensive care unit: Case studies with practical nursing solutions
}

\author{
Refaat A. Hegazi*, Mary Ann Cockram, Menghua Luo \\ Abbott Nutrition, Columbus, USA \\ Email: Refaat.Hegazi@abbott.com
}

Received 16 September 2012; revised 22 October 2012; accepted 5 November 2012

\begin{abstract}
The Guidelines for the Provision and Assessment of Nutrition Support Therapy in the Adult Critically Ill Patient published in May 2009 (joint guidelines between the Society of Critical Care Medicine (SCCM) and the American Society for Parenteral and Enteral Nutrition (A.S.P.E.N.) have advanced our clinical practice for the nutritional management of critically ill patients. In the current article, we will review how to implement these guidelines using a case study model. Two mechanically ventilated and tube fed patients are discussed, one with pneumonia and the second with severe acute pancreatitis. We address the questions of the feeding timing, method of administration, and management of its complications for these two patients.
\end{abstract}

Keywords: Enteral Nutrition; Critically Ill Patients; Guidelines; Case Studies

\section{INTRODUCTION}

Critical illness is often associated with catabolic stress and the systemic inflammatory response, which can lead to complications of increased infectious morbidity, multiorgan dysfunction, prolonged hospitalization, and increased mortality [1]. Critical illness is also associated with loss of lean body mass, which negatively impacts survival in the intensive care unit (ICU) and post discharge.

The stress response to critical illness can be modulated nutritionally with the use of early enteral nutrition, appropriate macro- and micronutrient delivery, and adequate glycemic control. A proactive therapeutic strategy of delivering early nutrition support therapy can help lessen disease severity, reduce complications, mitigate inflammation-related catabolism of lean body mass, decrease length of stay in the ICU, favorably modulate the

${ }^{*}$ Corresponding author. immune response [1,2], and positively impact patient outcomes. Providing early enteral nutrition within 24 48 hours of admission has been shown to reduce hospital length of stay [3,4], reduce ICU and hospital mortality [5,6], and reduce infectious complications [3,7].

To help guide clinical practitioners in prescribing and administering appropriate nutritional therapy in adult critically ill patients, the American Society for Parenteral and Enteral Nutrition (A.S.P.E.N.) and the Society of Critical Care Medicine (SCCM) published joint guidelines in May 2009 [1]. Recommendations of the Guidelines are given based on the number and level of evidence available in the literature. For example, the strongest recommendation is a Grade A recommendation, which is supported by at least two level I large, randomized trials. The Grade E recommendation is supported by nonrandomized studies, studies compared with historical controls, case series, uncontrolled studies, or expert opinion.

In the following review we will discuss these guidelines and apply them in clinical practice using a case study model. Through discussing two case studies, the current article presents common clinical issues that arise during enteral feeding of the critically ill patients. We will also review the evidence-supported practice guidelines of how to address these issues in order to prevent unnecessary interruption of enteral feeding. The experience and learning from these two cases could serve as a clinical tool to be generally used when enterally feeding the critically ill patients.

\section{PATIENT CASE 1}

A 55-year-old male presents to the emergency department with shortness of breath, productive cough of thick green sputum, wheezing and general malaise for the past 3 days. His vital signs are: Blood pressure 122/70, Heart rate 100 , Temperature $100.4^{\circ} \mathrm{F}$, Respiratory rate 24 .

The patient is admitted to the hospital and sputum is sent for culture and sensitivity. Chest X-ray indicates right lower lobe pneumonia. The patient is admitted to 
the medical unit for monitoring and treatment of pneumonia.

Twelve hours after admission the patient's respiratory status further declines and he is transferred to the ICU. The patient has hypoxemia, leukocytosis, and respiratory acidosis (Table 1). He is mechanically ventilated and vasopressors and propofol were started at this time.

\subsection{How Soon after Admission to the Hospital Should Feeding Be Started?}

We can look to the literature and guidelines to answer this question. In a study by Artinian and colleagues in 2006 [6] they retrospectively analyzed prospectively collected ICU database where they looked at the association of early enteral feeding and outcome in critically ill mechanically-ventilated patients. This was a large study that included more than 4000 patients. Based on when they received enteral nutrition relative to mechanical ventilation onset, patients were classified into 2 groups: early feeding (within 48 hours) $(n=2537)$ or the late feeding ( $n=1512$ ). The primary outcome of this study was both ICU and hospital mortality. Results show that patients who were fed within 48 hours of mechanical ventilation had less ICU and hospital mortality than the late feeding group (18.1\% vs $21.4 \%, P=0.01 ; 28.7 \%$ vs $33.5 \%, P=0.001$, respectively). This suggests that early feeding (within 48 hours) is associated with lower mortality. This early feeding is endorsed by the A.S.P.E.N. and SCCM guidelines advocating that enteral feeding should be started early within 24 - 48 hours of admission [1].

For our case study patient, the recommendation is to start enteral nutrition within the first 24 - 48 hours of admission assuming that the gastrointestinal (GI) tract is functioning. This question of functioning GI tract should be addressed early on when we see or care for ICU patients. The presence of bowel sounds is not the sole indication for starting tube feeding. Assessing GI function should also include medical history and physical examination findings.

\subsection{Why Should We Start Feeding Early?}

Studies have shown that early initiation of enteral feeding is associated with decreased infectious complications, shorter length of stay in the ICU, and some evidence of

Table 1. Admitting laboratory results of Case 1 patient.

\begin{tabular}{cccc}
\hline WBC $(\times \mathbf{1 0} / \mathbf{m L})$ & $\mathbf{H g b}(\mathbf{g} / \mathbf{d L})$ & Hct $(\%)$ & $\mathbf{p H}$ \\
14,000 & 11.5 & 42 & 7.31 \\
\hline $\mathbf{P a O}_{2}(\mathbf{m m H g})$ & $\mathbf{S a O}_{2}(\%)$ & $\mathbf{P a C O}_{2}(\mathbf{m m H g})$ & $\mathbf{H C O}_{3}(\mathbf{m m o l} / \mathbf{L})$ \\
64 & 76 & 56 & 24 \\
\hline
\end{tabular}

reduced mortality, as compared to late feeding [1].

\subsection{Why Should We Feed Enterally?}

To answer this question, we can review the A.S.P.E.N./ SCCM guidelines for feeding critically ill patients [1]. In patients with a functioning GI tract (e.g., patients with severe acute pancreatitis) the recommendation is that enteral nutrition (EN) is the preferred route of feeding over parenteral nutrition (PN). Of note, there are, however, some patients who have a non-functioning GI tract who will require nutrition support therapy with $\mathrm{PN}$.

A meta-analysis included in the Canadian clinical practice guidelines looked at studies that compared EN versus $\mathrm{PN}$ on infectious complications in critically ill patients [8]. The pooled effect of these studies is that EN significantly reduces the risk of infection and length of stay in ICU patients. The cost associated with EN is much less than that of PN with an estimated reduction of approximately $\$ 4000$ dollars per patient [9].

For our case study patient, the recommendation, therefore, is to start enteral feeding because it is associated with less infectious complications and improved clinical outcomes. Physiologically, enteral feeding preserves gut function because it is essential for the optimum functioning of the gut associated lymphoid tissue, a major immune system in our body that depends on a trophic effect of nutrients. Immune function is supported by enteral feeding.

\subsection{Should We Start Feeding at Goal Rate or Start at a Lower Rate?}

The recommendation is to start at a low rate (e.g., 20 - 30 $\mathrm{mL} / \mathrm{hr}$ ) and assess for GI tolerance allowing for gut acclimatization of the intestinal mucosa to the enteral feeding. This rate of enteral feeding is used for a reasonable period of time; (e.g., 8 - 12 hours) however, sometimes this range is more if the patient is hemodynamically unstable and we want to start with trophic feeding for a while, before advancing the feeding to goal rate. The decision to advance to goal rate depends on patient tolerance and the hemodynamic condition of the patient.

\subsection{This Patient Is on Vasopressors; Can We Still Feed This Patient Enterally?}

Each patient should be assessed individually, asking how severe is the hemodynamic instability, how many vasopressors and what doses is the patient on and whether these doses are escalating. In general, trophic feeding in this clinical setting will not be an absolute contraindication as long the patient's clinical and nutritional conditions are closely monitored by the clinical team (e.g., hemodynamic status, GI tolerance, and functional effects of trophic feeding) to best decide whether trophic feed- 
ing should be advanced to goal depending on the patient's condition. In a 2010 study, Khalid and colleagues [5] retrospectively reviewed the association of ICU and hospital mortality with early enteral feeding (within 48 hours) in patients who were on vasopressors (by definition, these patients were hemodynamically unstable). They found that early EN was associated with lower ICU and hospital mortality as compared with late EN (22.5\% vs $28.3 \%, P=0.03$; $33.8 \%$ vs $43.9 \%, P<0.001$, respectively).

In summary, the learning points from this patient case are:

- Early enteral feeding of critically ill patients is recommended based on the A.S.P.E.N./SCCM evidencebased guidelines [1].

- Enteral feeding is associated with more favorable outcomes compared to parenteral feeding.

- Assessing patients' GI function and tolerance to enteral formulations helps patients meet nutritional requirements and minimize any GI complications associated with starting enteral feeding.

\section{PATIENT CASE 2}

A 58-year-old obese (BMI 36) female presents to the emergency department complaining of 3 days of severe abdominal pain which radiates to the back, with nausea and vomiting. Her vital signs are: Blood pressure 88/48; Heart rate 132 ; Temperature $102.0^{\circ} \mathrm{F}$; Respiratory rate 34 . See Table 2 for laboratory values.

Pancreatic enzymes are elevated and the CT of the abdomen reveals there is a large pseudocyst that is compressing the gastric outlet causing obstruction and peripancreatic edema. She is identified as having severe acute pancreatitis. The triglycerides are elevated; there is some evidence of kidney dysfunction, and mild elevation of liver enzymes.

By reviewing white blood cell (WBC) count, vital signs, and the diagnosis of severe acute pancreatitis, we can identify that the patient has systemic inflammatory response syndrome. With her progressive course of hypoxemia, the patient is started on mechanical ventilation.

Table 2. Admitting laboratory results of Case 2 patient.

\begin{tabular}{cccc}
\hline $\begin{array}{c}\text { WBC } \\
(\times \mathbf{1 0} / \mathbf{m L}) \\
14,000\end{array}$ & Hct (\%) & $\begin{array}{c}\text { Glucose } \\
(\mathbf{m g} / \mathbf{d L})\end{array}$ & BUN (mg/dL) \\
\hline $\begin{array}{c}\text { Creatinine } \\
(\mathbf{m g} / \mathbf{d L})\end{array}$ & $\begin{array}{c}\text { Amylase } \\
(\mathbf{I U} / \mathbf{L})\end{array}$ & Lipase (IU/L) & AST (IU/L) \\
2.1 & 590 & 1400 & 67 \\
\hline ALT (IU/L) & $\begin{array}{c}\text { Bilirubin } \\
(\mathbf{m g} / \mathbf{d L})\end{array}$ & INR & $\begin{array}{c}\text { Triglycerides } \\
(\mathbf{m g} / \mathbf{d L})\end{array}$ \\
55 & 1.1 & 1.2 & 380 \\
\hline
\end{tabular}

\subsection{This Patient Is Obese; Can She Tolerate Being Kept NPO?}

To answer this question, we need to look at the new definition of malnutrition integrating the inflammatory process in the diagnosis of malnutrition. According to Jensen et al. [10] one needs to assess nutrition risk, does the patient have low food intake or loss of lean body mass. Next, we need to evaluate whether inflammation is present. If inflammation is present, is it mild-moderate or severe. Patients with chronic disease, such as chronic kidney disease, cancer, rheumatoid arthritis, or sarcopenic obesity, by definition are malnourished with mild to moderate inflammation. If inflammation is severe as encountered in sepsis, burns, and trauma it is diagnosed as acute disease-related malnutrition.

The patient in the case study is malnourished, because she has manifestation of systemic inflammatory response syndrome and meets the diagnostic criteria of acute severe inflammation-associated malnutrition.

The answer to the question then is no, she should not be kept NPO. Obese patients are not over-nourished or even well-nourished. Sarcopenic obesity (loss of muscle mass or strength) commonly seen in critically ill obese patients complicates the critical illness. Weight loss at this time should not be a goal for this ICU patient. We need to distinguish between the obese individual with preserved muscle mass and the critically ill obese person who needs to be fed in order to preserve lean body mass as well as to nutritionally stimulate gut associated lymphoid tissue.

\subsection{Can Patients with Severe Acute Pancreatitis Be Fed Enterally or Is Parenteral Nutrition (PN) the Best Choice for Feeding Patients with This Condition?}

In a meta-analysis published in 2004 by Marik and Zaloga [11], randomized controlled trials in patients with severe acute pancreatitis being fed EN or PN were analyzed to evaluate the effect of the type of feeding on infectious complications. Enteral nutrition in patients with severe acute pancreatitis was associated with lower incidence of infections, less surgical interventions, and length of hospital stay. Clearly, EN has benefits beyond calories and protein and plays a role in maintaining a physiological homeostatic function of the gut associated lymphoid tissue.

The recommendation for this patient is that EN is preferred over PN as a safe source of feeding that can decrease risk of infectious and surgical interventions and length of ICU stay. The decision to start PN will depend on the GI function of the patient and the adequacy of nutritional therapy by EN to meet the patient's increased nutritional needs. 


\subsection{When Should Enteral Feeding Be Started for This Patient?}

To answer this question, we can review a retrospective analysis of patients with severe acute pancreatitis who were treated according to an established protocol [12]. All patients were started on tube feeding within 24 hours of getting a consult with the same nasogastric-jejunal tube, and all patients were fed a peptide-based formula. In this analysis, we looked at the outcome of ICU length of stay and its association with the time from initiating tube feeding to the time to reach goal rate feeding. We found that patients who reached goal rate feeding in 3 or less days from the time of initiating feeding had shorter length of ICU stay as compared to patients who never reached goal rate of feeding. When we looked at APACHE II scores to see if the severity of illness was different between those patients who reached goal feeding in 3 days or less compared to those who never reached goal rate, no difference was found between the groups in severity of illness based on APACHE II scores. This suggests that the timing between initiating enteral feeding and reaching goal feeding is associated with better outcomes irrespective of the severity of disease. We also looked at the time from the onset of pain to the time to reach goal feeding rate. The patients who reached goal rate from the onset of pain earlier spent less time in the ICU. In conclusion the earlier we feed (within 24 hours) and the earlier we reach goal rate appears to be associated with better outcomes. Achievement of distal jejunal feeding goals early was associated with shorter ICU length of stay irrespective of severity of acute pancreatitis.

The recommendation is that enteral feeding should be started as early as the first 24 - 48 hours, assuming a functioning GI tract as early initiation of enteral feeding is associated with favorable clinical outcomes.

With our patient, a nasal jejunal tube was not able to be placed beyond the ligament of Treitz so a nasogastric tube was inserted at the bedside. A continuous feeding is started at $85 \mathrm{~mL} /$ hour via a feeding pump. The patient develops diarrhea and has a total of 6 loose stools over the next 24 hours. Gastric residual volumes (GRV) are being checked every 4 hours and are running between 250 and $300 \mathrm{~mL}$ at each 4-hour check.

\subsection{What Is Likely Contributing to the Diarrhea This Patient Is Experiencing? What Should We Do to Manage the Diarrhea?}

In a review of the relationship between tube-feeding initiation and diarrhea, studies have found that in up to $60 \%$ of the cases of tube-feeding associated diarrhea, the cause was not the tube feeding itself, it was other causes [13]. The two major causes were medications (e.g., sor- bitol-containing, antibiotics, proton pump inhibitors) and intestinal infections [14]. Before stopping the tube feeding, one should exclude other causes of diarrhea other than the enteral feeding and then to slow the rate and maintain for more than 12 hours, even maybe 24 hours, if necessary, to allow for gut acclimatization and then after enough period of time, slowly advance the rate. If the patient still does not tolerate the lower rate, we should consider changing the formula from a standard, intact protein formula, to a hydrolyzed, peptide-based formula that can help with improving the GI tolerance. If the patient is not responding to this change in formula type to a peptide-based formula, in some cases, the only solution is to switch the patient from enteral feeding to parenteral feeding.

\subsection{How Would You Manage a Patient Who Is Having GRV between 250 - 300 mL Every 4 Hours?}

The A.S.P.E.N./SCCM guidelines [1] recommend a GRV up to $500 \mathrm{~mL}$ without switching formulas or stopping the feeding. The guidelines advocate the use of promotility agents to help with gastric motility. The studies relating increased GRV and aspiration pneumonia are not consistently strong. Aspiration precautions should always be implemented, such as raising the head of the bed $30^{\circ}$ $45^{\circ}$, and if there is a high risk of aspiration, use a duodenal or postpyloric feeding instead of gastric feeding. By using aspiration precautions one should not have to worry about GRV, if a promotility agent is used and the patient is monitored.

To summarize points discussed with this patient case:

- Early enteral feeding is recommended for ICU patients as well as patients with severe acute pancreatitis according to the A.S.P.E.N./SCCM guidelines [1].

- Diarrhea in tube fed patients is most often caused by factors other than the tube feeding (exclude other factors, adjust tube feeding rates before stopping the feeding or switching formula types).

- GRV is an important factor to monitor. A value of GRV up to $500 \mathrm{~mL}$ may be acceptable according to the A.S.P.E.N./SCCM guidelines [1]. Use of prokinetic agents can help with gastric emptying. Aspiration precautions should be used in all ICU patients.

In summary, the integration of the evidence-based practice guidelines in the nutritional management of clinical issues commonly faced when enterally feeding patients in the ICU could help improve clinical outcomes by optimizing the nutritional status of the patients via preventing unnecessary stopping of tube feeding. Unwarranted interruption of enteral feeding should be discouraged and every effort should be made to maintain and improve the nutritional status of critically ill patients for 
better clinical outcome.

\section{ACKNOWLEDGEMENTS}

R.A. Hegazi, M.A. Cockram and M. Luo are full time employees of Abbott Laboratories. The material presented in this article is based on the best-known clinical evidence and is not affected by this financial relationship.

\section{REFERENCES}

[1] McClave, S.A., Martindale, R.G., Vanek, V.W., McCarthy, M., Roberts, P., Taylor, B., Ochoa, J.B., Napolitano, L. and Cresci, G. (2009) Guidelines for the provision and assessment of nutrition support therapy in the adult critically ill patient: Society of Critical Care Medicine (SCCM) and American Society for Parenteral and Enteral Nutrition (A.S.P.E.N.). Journal of Parenteral and Enteral Nutrition, 33, 277-316. doi:10.1177/0148607109335234

[2] Wischmeyer, P.E. and Heyland, D.K. (2010) The future of critical care nutrition therapy. Critical Care Clinics, 26, 433-441. doi:10.1016/j.cc. 2010.04.011

[3] Kattelmann, K.K., Hise, M., Russell, M., Charney, P., Stokes, M. and Compher, C. (2006) Preliminary evidence for a medical nutrition therapy protocol: Enteral feedings for critically ill patients. Journal of the American Dietetic Association, 106, 1226-1241. doi:10.1016/j.jada.2006.05.320

[4] Andersen, H.K., Lewis, S.J. and Thomas, S. (2006) Early enteral nutrition within $24 \mathrm{~h}$ of colorectal surgery versus later commencement of feeding for postoperative complications. Cochrane Database of Systematic Reviews, CD004080.

[5] Khalid, I., Doshi, P. and DiGiovine, B. (2010) Early enteral nutrition and outcomes of critically ill patients treated with vasopressors and mechanical ventilation. American Journal of Critical Care, 19, 261-268. doi:10.4037/ajcc2010197

[6] Artinian, V., Krayem, H. and DiGiovine, B. (2006) Effects of early enteral feeding on the outcome of critically ill mechanically ventilated medical patients. Chest, 129, 960-967. doi:10.1378/chest.129.4.960
[7] Heyland, D.K., Dhaliwal, R., Drover, J.W., Gramlich, L. and Dodek, P. (2003) Canadian clinical practice guidelines for nutrition support in mechanically ventilated, critically ill adult patients. Journal of Parenteral and Enteral Nutrition, 27, 355-373. doi:10.1177/0148607103027005355

[8] 2009 Clinical Practice Guidelines. The Use of EN vs PN, 8 October 2012

http://www.criticalcarenutrition.com/docs/cpg/1.0envspn 07 FINAL.pdf.

[9] Cangelosi, M.J., Auerbach, H.R. and Cohen, J.T. (2011) A clinical and economic evaluation of enteral nutrition. Current Medical Research and Opinion, 27, 413-422. doi:10.1185/03007995.2010.545816

[10] Jensen, G.L., Mirtallo, J., Compher, C., Dhaliwal, R., Forbes, A., Grijalba, R.F., Hardy, G., Kondrup, J., Labadarios, D., Nyulasi, I., Castillo Pineda, J.C. and Waitzberg, D. (2010) Adult starvation and disease-related malnutrition: A proposal for etiology-based diagnosis in the clinical practice setting from the International Consensus Guideline Committee. Journal of Parenteral and Enteral Nutrition, 34, 156-159. doi:10.1177/0148607110361910

[11] Marik, P.E. and Zaloga, G.P. (2004) Meta-analysis of parenteral nutrition versus enteral nutrition in patients with acute pancreatitis. British Medical Journal, 328, 1407. doi:10.1136/bmj.38118.593900.55

[12] Hegazi, R., Raina, A., Graham, T., Rolniak, S., Centa, P., Kandil, H. and O’Keefe, S.J. (2011) Early jejunal feeding initiation and clinical outcomes in patients with severe acute pancreatitis. Journal of Parenteral and Enteral Nutrition, 35, 91-96. doi:10.1177/0148607110376196

[13] Edes, T.E., Walk, B.E. and Austin, J.L. (1990) Diarrhea in tube-fed patients: Feeding formula not necessarily the cause. The American Journal of Medicine, 88, 91-93. doi:10.1016/0002-9343(90)90454-L

[14] Btaiche, I.F., Chan, L.N., Pleva, M. and Kraft, M.D. (2010) Critical illness, gastrointestinal complications, and medication therapy during enteral feeding in critically ill adult patients. Nutrition in Clinical Practice, 25, 32-49. doi:10.1177/0884533609357565 